\section{Contemporary perspective on plaque control}

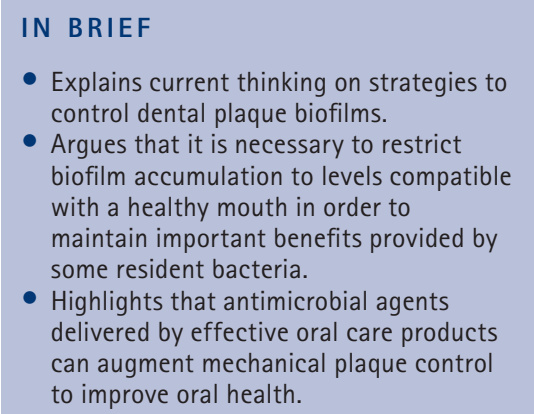

- Explains current thinking on strategies to control dental plaque biofilms.

Argues that it is necessary to restrict . with a healthy mouth in order to some resident bacteria.

P. D. Marsh ${ }^{1}$

VERIFIABLE CPD PAPER

The aim of this review article is to provide a scientific platform that will enable the dental team to develop a rational approach to plaque control based on the latest knowledge of the role of the oral microflora in health and disease. The resident oral microflora is natural and forms spatially-organised, interactive, multi-species biofilms on mucosal and dental surfaces in the mouth. These resident oral microbial communities play a key function in the normal development of the physiology of the host and are important in preventing colonisation by exogenous and often undesirable microbes. A dynamic balance exists between the resident microflora and the host in health, and disease results from a breakdown of this delicate relationship. Patients should be taught effective plaque control techniques that maintain dental biofilms at levels compatible with oral health so as to retain the beneficial properties of the resident microflora while reducing the risk of dental disease from excessive plaque accumulation. Antimicrobial and antiplaque agents in oral care products can augment mechanical plaque control by several direct and indirect mechanisms that not only involve reducing or removing dental biofilms but also include inhibiting bacterial metabolism when the agents are still present at sub-lethal concentrations.

\section{INTRODUCTION}

Dental professionals are faced with a number of apparent paradoxes when it comes to advising patients on the most appropriate strategy for plaque control. During training, students are taught that caries and periodontal diseases result from the activity of bacteria in dental plaque and that effective self-performed plaque removal is an essential part of the prevention and management of these diseases; but now it is being reported that many of the micro-organisms in the mouth make an important contribution to our well-being! Also, many plaque control products are formulated with antimicrobial agents that are described as being broad spectrum and yet these products also have to meet the regulatory guidelines that demand that they do not disrupt the natural balance of the normal oral microflora. In the face of these apparently contradictory views and

'Department of Oral Biology, Leeds Dental Institute, University of Leeds, Clarendon Way, Leeds, LS2 9LU, UK Correspondence to: Professor Philip Marsh

Email: p.d.marsh@leeds.ac.uk; Tel: +44 (0)1980 612287

\section{Refereed Paper}

Accepted 4 April 2012

DOI: 10.1038/sj.bdj.2012.524

${ }^{\circ}$ British Dental Journal 2012; 212: 601-606 requirements, how does the dental team decide on a rational way forward? The aim of this article is to briefly review the current knowledge in this area, give answers to some common questions and provide the evidence to support a contemporary approach to plaque control.

\section{WHAT IS THE RELATIONSHIP BETWEEN HUMANS AND THEIR NATURAL MICROFLORA?}

It is a remarkable statistic that humans are made up of about $10^{14}$ cells $^{1}$ of which only ten percent are mammalian. The majority are the micro-organisms (the natural resident microflora) that inhabit all environmentally-exposed surfaces of the body, where they form biofilms. ${ }^{2}$ The composition of these biofilms varies at distinct sites around the body and is directly influenced by the biological and physical conditions associated with the particular habitat. These biofilms are formed of symbiotic communities of different microorganisms that grow on, and interact with, the surfaces they colonise. Biofilms develop in a structured way, are spatiallyand functionally-organised, and the constituent species communicate and interact with one another. ${ }^{3}$ These polymicrobial biofilms display novel properties; of clinical relevance is that they are much less susceptible to the host defences and antimicrobial agents. ${ }^{4}$ The reasons for this are still the subject of much debate, but are directly linked to the properties of the biofilm itself, since the organisms retain their intrinsic sensitivity if the biofilm is dispersed. The most common explanations for the reduced susceptibility of biofilms to antimicrobial agents include:

- Reduced penetration of the agent into the biofilm or quenching of the agent at the surface of the biofilm

- The novel properties expressed by bacteria when growing on a surface

- Sub-optimal conditions for activity

- The slow growth rates of attached bacteria within biofilms.

The resident microflora has evolved to co-exist in harmony with the host and carry out key functions that are essential to our well-being. These functions include the ability to prevent colonisation by exogenous (and often pathogenic) microorganisms (a process termed colonisation resistance), and in the normal development of the physiology, nutrition and immune system of the host., ${ }^{2,5}$ 
The mouth is similar to other sites in the body in that it has a natural microflora with a characteristic composition that confers benefit (see later). ${ }^{6}$ However, on occasions, this beneficial relationship can break down and disease can occur (for example, dental caries, periodontal diseases), while halitosis can also be a consequence.

Therefore, it is essential to appreciate the benefits that are derived from a balanced relationship between the oral microflora and the host and to understand the processes that predispose a site to disease if effective control and preventative measures are to be adopted. Of clinical relevance is that these measures might vary from person to person.

\section{WHAT BENEFITS DO THE RESIDENT ORAL MICROFLORA PROVIDE TO THE HOST?}

The mouth is well equipped with an array of host defences provided by both the innate and adaptive arms of the immune system and yet all mucosal and dental surfaces are naturally colonised by a diverse collection of micro-organisms. It is becoming clear that the host is not indifferent to the presence of these consortia of microbes and has developed mechanisms that permit a beneficial relationship. ${ }^{7}$ There is evidence for active communication ('crosstalk') between some of the resident bacteria and mucosal cells that downregulates potentially damaging pro-inflammatory host responses to the normal oral microflora, while the host retains the ability to respond to genuine microbial insults. ${ }^{78}$ The precise biological mechanisms involved in this 'cross-talk' are still being determined, but pathogenic and non-pathogenic bacteria may initiate different intracellular signalling pathways and innate immune responses in epithelial cells. ${ }^{9}$

As at other body sites, the resident oral microflora displays 'colonisation resistance' and prevents the establishment in the mouth of the many exogenous microorganisms we come into contact with on a daily basis. This is because the natural oral microflora is better adapted at attachment to oral surfaces, is more efficient at metabolising the available nutrients for growth and can produce inhibitory factors and create hostile environments that restrict colonisation by potential microbial invaders. A consequence for patients on long-term broad spectrum antibiotic treatment is that the resident oral microflora can be suppressed resulting in overgrowth by yeasts and environmental bacteria in the mouth.

Recent findings suggest that the resident oral bacteria contribute to the maintenance of healthy gastrointestinal and cardiovascular systems via the metabolism of dietary nitrate. Approximately 25\% of ingested nitrate is secreted in saliva where some oral resident bacteria reduce nitrate to nitrite. Nitrite can affect a number of key physiological processes including the regulation of blood flow, blood pressure, gastric integrity and tissue protection against ischemic injury. Nitrite can be further converted to nitric oxide in the acidified stomach, and this has antimicrobial properties, and contributes to defence against enteropathogens and in the regulation of gastric mucosal blood flow and mucus formation. The reduction of nitrate to nitrite in saliva fell markedly in human volunteers, ${ }^{10-12}$ and laboratory animals, ${ }^{11}$ when the resident salivary microflora was deliberately suppressed using antimicrobial agents. The suppression of endogenous nitrate reduction in the animal model resulted in a loss of the predicted biological benefits of nitrite, including reduced gastric mucus thickness, while the expected fall in blood pressure following a nitrate supplement was prevented. ${ }^{11}$

It is of clinical importance, therefore, that oral antimicrobials are applied according to the recommended instructions which aim to maintain the microflora of the mouth at levels that are compatible with oral health, but below those which are associated with disease. This is in order to preserve the beneficial functions of these important resident microbes which, it is becoming clear, are essential for both the general and oral health of that person. Thus, antibiotics are not a recommendation for managing chronic periodontal disease. ${ }^{13}$

\section{CAN WE DEFINE WHAT IS 'NORMAL' IN TERMS OF OUR ORAL MICROFLORA?}

It is surprisingly difficult to fully define what might be regarded as the normal, resident oral microflora. At present, only about $50 \%$ of the oral microflora can be cultivated in the laboratory. This is because of our ignorance of the growth requirements of the more fastidious members of the oral microflora, but also due to our naivety in attempting to grow bacteria as pure cultures in the laboratory when they have evolved to grow in oral biofilms as consortia and interact closely with neighbouring species with complementary properties. ${ }^{14}$ The application of cultureindependent, molecular approaches has identified about 1,200 different types of microbe that can inhabit the human mouth. ${ }^{1}$ However, any particular mouth may contain only up to about 80 species, ${ }^{15}$ although the application of more powerful and sensitive molecular approaches will increase this number, as species that are present only in low numbers will be detected. The Human Oral Microbiome project is underway and aims to identify and characterise all members of the resident oral microflora; ${ }^{1}$ the conclusion of these studies will permit a more accurate description of what is the "normal oral microflora'. Information is being placed in a publically accessible web-based Human Oral Microbiome Database (http://www. homd.org), which also feeds information into the larger Human Microbiome project.

The normal oral microflora is diverse and varies in composition between sites due to differences in the prevailing biological conditions. ${ }^{16}$ The load on mucosal sites is low due to desquamation. In contrast, teeth (being non-shedding surfaces) potentially permit the accumulation of large masses of bacteria and their products, especially at stagnant or 'difficult-toreach' areas, unless effective oral hygiene is practised. The microbial composition of oral biofilms varies depending on the site or surface, because local environmental conditions dictate which organisms are able to colonise, grow and be either major or minor components of the established microbial community. For example, the bacteria found in occlusal fissures are mainly Gram positive (especially streptococci), are facultatively anaerobic and metabolise host and dietary sugars, and the site is affected by the properties of saliva. In contrast, the biofilms from the healthy gingival crevice contain many Gram negative and obligately anaerobic species, that have a proteolytic style of metabolism, and the community is influenced by gingival crevicular fluid (GCF), a serum-like exudate. ${ }^{16}$ Certain bacteria are 
commonly found in high proportions at healthy sites and can be regarded as part of the core resident oral microflora. These include members of the bacterial genera: Streptococcus, Actinomyces, Neisseria, Haemophilus, Veillonella, Prevotella and Fusobacterium, but the individual species and their proportions may vary between sites and between people.

One of the challenges in defining what is 'normal' in terms of the resident oral microflora is that, traditionally, comparisons have been made from different studies of 'lists' of bacterial names. In diverse biofilms, such as those in the oral cavity, this may not be an appropriate approach. Within any microbial community, bacteria will have a particular role or function (for example, the catabolism of complex host glycoproteins to individual sugars and peptides; proteolysis of host proteins and peptides to simpler peptides and amino acids respectively; consumption of oxygen to create a more anaerobic environment etc). Organisms with different 'names' could carry out equivalent roles or activities, so that the definition and description of the resident oral microflora should be based around functional characteristics rather than simply by bacterial name.

The composition of the oral microflora can remain stable over time (microbial homeostasis). ${ }^{17}$ This is not due to any biological indifference among the members of the biofilm community - the relationship is not passive but highly dynamic. Biofilm composition will respond to changes in local environment (for example, in saliva flow, status of host defences, etc) and lifestyle (diet, smoking, etc). Such changes can perturb biofilm composition and activity and predispose a site to disease. Oral diseases are generally associated with shifts in the balance of the microflora at a site, so previously minor members of the biofilm become predominant, and the overall metabolic activity of the biofilm changes. ${ }^{18}$ Thus, in contrast to many situations in medical microbiology, it is too simplistic to talk of the presence of 'good' or 'bad' bacteria. Disease is a result of undesirable changes to the microbial balance, metabolism, and composition of these dental biofilms.

As we shall see, of clinical relevance is the need to not only apply appropriate plaque control strategies to reducing dental disease, but to also try to identify and remedy the factors that drive these deleterious changes in the microbiological composition and metabolism of these biofilms.

\section{SO WHAT IS THE PURPOSE OF PLAQUE CONTROL?}

Patients need to maintain plaque at levels compatible with health in order to prevent the breakdown of microbial homeostasis which would increase the risk of disease. It is inappropriate and futile for patients and dental professionals to attempt to eliminate plaque biofilms; rather, patients should be using effective oral hygiene practices, combined with an appropriate lifestyle, to try to control plaque at levels compatible with health so as to maintain the beneficial properties of the resident oral microflora, and reduce the risk of disease. In periodontitis, plaque control 'thresholds' that are compatible with a health-promoting biomass vary from patient-to-patient, with some requiring extremely good plaque control, while others manage with less stringent regimes.

\section{WHICH MICRO-ORGANISMS CAUSE DENTAL DISEASE?}

Dental diseases are associated with an imbalance in the composition of the resident oral microflora. ${ }^{18}$ Disease is linked to the presence of higher proportions of certain species that are normally only minor components in the biofilm. In dental caries, demineralisation is associated with increased proportions of mutans streptococci, lactobacilli and bifidobacteria. The virulence traits are relatively nonspecific and centred around sugar metabolism, such as the ability of these bacteria to rapidly transport sugars into the cell and metabolise them to acid, and then to survive and grow under the conditions of low $\mathrm{pH}$ generated within the biofilm (acid tolerance). The ability to synthesise intracellular and extracellular polysaccharides from sucrose also plays a role in developing a cariogenic biofilm. In gingivitis, there is an increase in plaque mass, which provokes an inflammatory response by the host. If unresolved, by-stander damage to the periodontium can occur from an inappropriate and exaggerated host response to subgingival bacteria and their metabolites. Many of the implicated bacteria are currently unculturable; others are Gram negative, obligately anaerobic and highly proteolytic. Virulence traits of these bacteria include the production of proteases, cytotoxins and inflammatory mediators.

Biofilms can form on mucosal surfaces, and substantial numbers of bacteria can be found on the tongue. In some subjects, this can result in halitosis, which is also linked to the metabolism of obligately anaerobic, proteolytic bacteria resulting in the production of volatile sulphur and other malodorous compounds. ${ }^{19,20}$

Evidence linking oral and general health is accumulating, particularly with respect to diabetes mellitus and cardiovascular and respiratory diseases. Some studies also report a weak positive association between periodontal disease and adverse pregnancy outcomes. ${ }^{21}$ The hypothesis behind the link between oral and general health is that many oral bacteria act as opportunistic pathogens, especially if they enter the blood stream and reach sites not normally accessible to them (including heart valves and atheromatous plaques); or if the host defences are compromised and subgingival biofilms in periodontal disease contain bacteria which (a) express inflammatory cell surface components (for example, lipopolysaccharide) and (b) shed metabolites which induce prostaglandins and inflammatory mediators. The vascular nature of the periodontium means that these pro-inflammatory mediators can affect distant sites in the body. Oral microorganisms may also give rise to aspiration pneumonia in susceptible patients as anaerobic bacteria from periodontal pockets have been isolated from infected lungs. ${ }^{22}$ This is another way in which effective oral hygiene can contribute to maintaining the general health of an individual.

\section{HOW CAN EFFECTIVE PLAQUE CONTROL BE ACHIEVED?}

As discussed earlier, dental plaque preferentially accumulates at stagnant sites on teeth that many individuals find difficult to clean- these sites are also the most disease susceptible. Mechanical plaque control can be effective, but needs to be meticulous and patients have to be highly motivated and with an appropriate lifestyle (that is, an appropriate diet, avoid smoking, etc). Consequently, oral care products have been formulated that contain antiplaque or 
antimicrobial agents to augment conventional mechanical plaque control activities and interfere with biofilm composition and metabolism, especially at sites that are difficult to clean and are commonly missed during self-performed mechanical plaque control.

Antiplaque agents function by removing or disrupting biofilms, or by preventing the formation of new biofilm, without necessarily killing the component microorganisms. In contrast, antimicrobial agents inhibit the growth of (bacteriostatic action) or kill (bactericidal action) microorganisms in oral biofilms and are defined in terms of the minimum inhibitory concentration (MIC) or minimum bactericidal concentration $(\mathrm{MBC})$ respectively. ${ }^{23}$ The activity of these agents can be against a limited (narrow spectrum) or wider (broad spectrum) target group of micro-organisms. The mode of action of antimicrobial agents is influenced by their concentration and the length of time they are in contact with the target organisms. Typically, the MIC/MBC of an agent is determined in the laboratory on liquid grown (planktonic) cells in tests where the agent is in contact with a pure culture of the organism for prolonged periods (24-48+ hours). However, as discussed above, bacteria growing on a surface as a biofilm show reduced sensitivity to killing by antimicrobial agents, especially in older (more mature) biofilms. Moreover, the maximum length of time recommended for people to brush their teeth is in the order of two minutes, followed by flossing and rinsing with a mouthwash for 30-60 seconds. A major requirement of the antiplaque formulation, therefore, is to deliver sufficient concentration of the active ingredients to have an effect on the biofilm in that short period of time. Alternatively, the formulation should ensure the prolonged retention of the active components on dental and mucosal surfaces in the mouth so that they can be released over time at levels that will still deliver biological activity.

An example of the pharmacokinetic profile of a representative antimicrobial agent delivered to the mouth from an oral care product is shown in Figure 1. There are only short periods where the agent is present at a high concentration (that is, at concentrations greater than the MIC or $\mathrm{MBC}$ ) followed by longer periods where

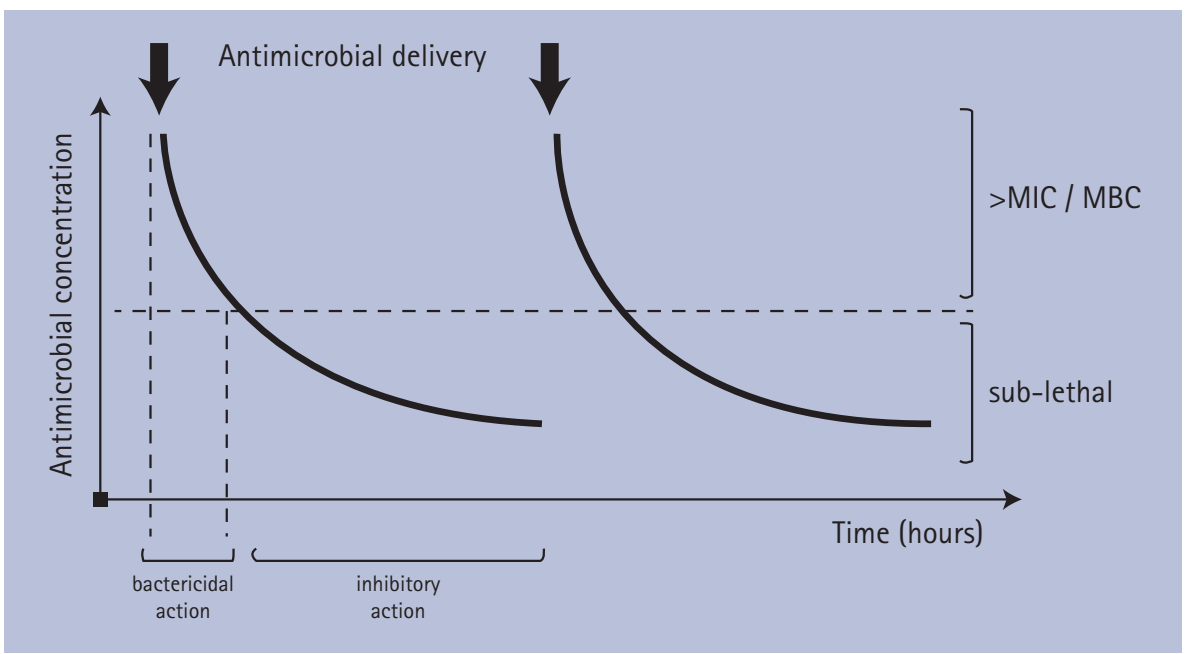

Fig. 1 Pharmacokinetics of antimicrobial agents delivered to the mouth. ${ }^{23} \mathrm{~A}$ schematic representation of the change in concentration over time following the delivery to the mouth on two occasions of an antimicrobial agent from an oral care product. The agent may be present above its MIC/MBC level for a relatively short period before it is lost from the mouth. The agent may be present for longer at sub-lethal concentrations; agents may still exert beneficial effects by inhibiting traits associated with bacterial pathogenicity (see Table 1). The dynamics of the curve will vary for each antimicrobial agent)

\begin{tabular}{|c|c|c|}
\hline Class of inhibitor & Example & Antimicrobial action at sub-lethal concentrations* \\
\hline Bisbiguanide & Chlorhexidine* & $\begin{array}{l}\text { Inhibits sugar transport and acid production } \\
\text { Inhibits amino acid uptake, polysaccharide synthesis } \\
\text { and bacterial membrane functions } \\
\text { Inhibits protease activity }\end{array}$ \\
\hline Enzymes & $\begin{array}{l}\text { Mutanase, dextranase, } \\
\text { amyloglucosidase- } \\
\text { glucose oxidase }\end{array}$ & $\begin{array}{l}\text { Degrade bacterial polysaccharides that } \\
\text { make up plaque biofilm matrix } \\
\text { Boosts salivary peroxidise system which } \\
\text { can inhibit bacterial glycolysis }\end{array}$ \\
\hline Essential oil extracts & $\begin{array}{l}\text { Menthol, thymol, } \\
\text { eucalyptol, methyl } \\
\text { salicylate ** }\end{array}$ & $\begin{array}{l}\text { Inhibit acid production and bacterial growth } \\
\text { Reduces lipopolysaccharide }\end{array}$ \\
\hline Metal salts & $\begin{array}{l}\text { Zinc, copper, } \\
\text { stannous ions }\end{array}$ & $\begin{array}{l}\text { Inhibit sugar transport and acid production } \\
\text { Inhibit protease activity }\end{array}$ \\
\hline Natural molecules & $\begin{array}{l}\text { Plant extracts } \\
\text { (for example, } \\
\text { apigenin, } t t \text {-farnesol) }{ }^{-\cdots}\end{array}$ & $\begin{array}{l}\text { Inhibit acid production } \\
\text { Inhibit bacterial polysaccharide synthesis }\end{array}$ \\
\hline Phenols & Triclosan & $\begin{array}{l}\text { Inhibit sugar transport and acid production } \\
\text { Inhibit protease activity }\end{array}$ \\
\hline $\begin{array}{l}\text { Quaternary ammonium } \\
\text { compounds }\end{array}$ & $\begin{array}{l}\text { Cetylpyridinium } \\
\text { chloride* }^{* *}\end{array}$ & \\
\hline Surfactants & $\begin{array}{l}\text { Sodium lauryl } \\
\text { sulphate, delmopinol }\end{array}$ & $\begin{array}{l}\text { Damage cell membranes } \\
\text { Inhibit bacterial enzymes }\end{array}$ \\
\hline
\end{tabular}

the agent is present at sub-lethal levels. This profile has a significant influence on the mode of action of these agents. ${ }^{23}$ Organisms reported to have an apparent similar sensitivity to an antimicrobial agent (as determined in a standard MIC assay format under laboratory conditions) can vary markedly in their susceptibility when exposed to the agent for only relatively short periods, as occurs during routine oral use; and sometimes favourable selective inhibitory effects can be obtained. For example, although displaying similar MIC values against Triclosan, Gram negative obligately anaerobic bacteria implicated in gingivitis and periodontal diseases are more susceptible than the Gram positive bacteria (streptococci and Actinomyces species) found in health when exposed to this agent for only short periods. ${ }^{24}$ Many 


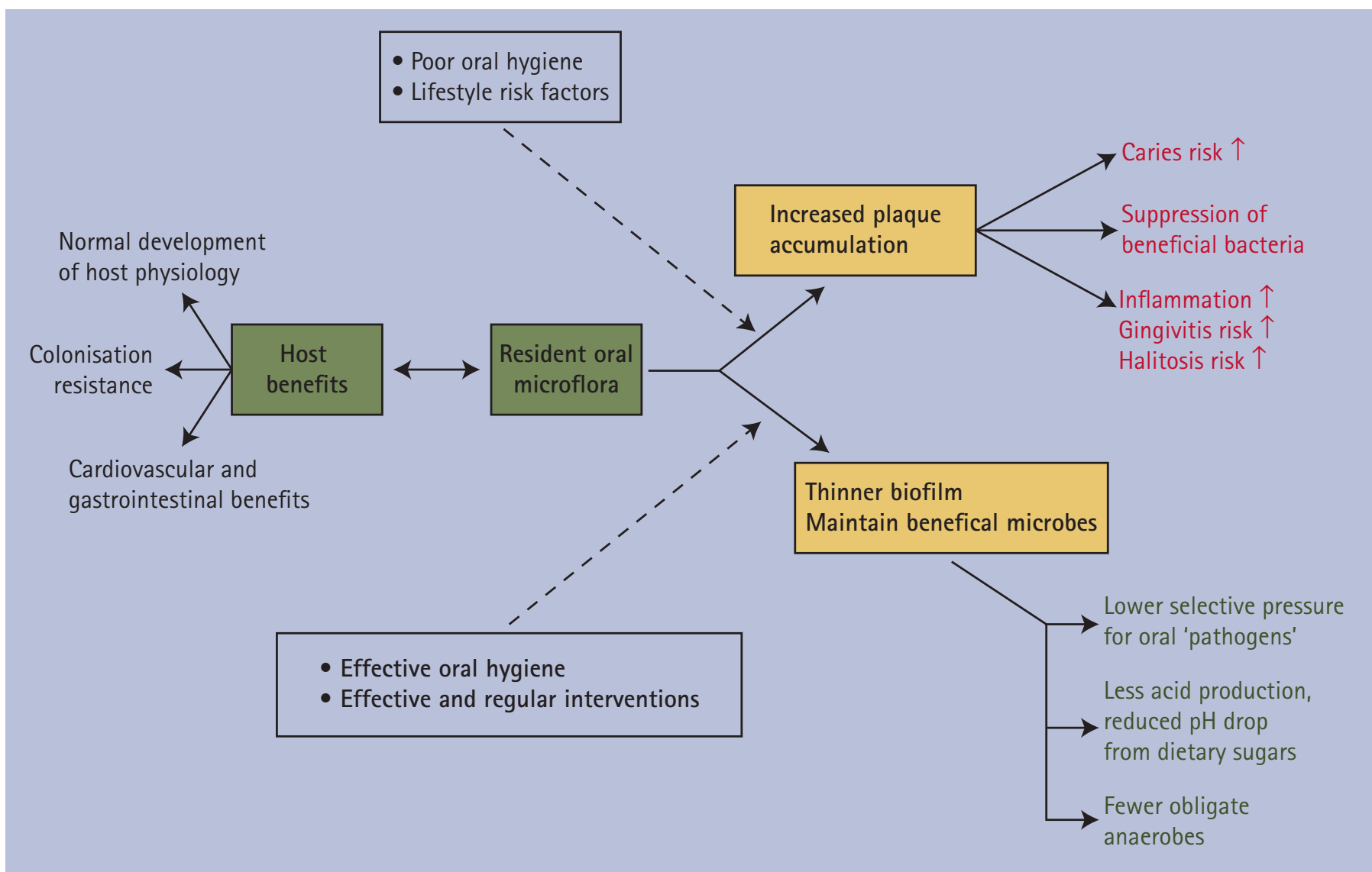

Fig. 2 The relationship between the resident oral microflora and the host in health and disease. The resident oral microflora is important for the normal development of many functions of the host in health, and contributes to the host defences (colonisation resistance). If plaque is allowed to accumulate then the patient is at risk of caries, gingivitis or halitosis. Effective plaque control should maintain the oral microflora at levels that are compatible with health so as to retain the beneficial properties of the resident oral microflora, while minimising the risk of disease

active agents used in oral care products are able to exert a clinically relevant effect when present below the MIC/MBC by inhibiting the expression of virulence traits by oral bacteria, such as sugar transport mechanisms, acid production, extracellular polysaccharide synthesis and protease activity (Table 1). ${ }^{23}$ In this way, small but regular (for example, twice daily) subtle and minor inhibitory effects on the plaque biofilm can:

- Reduce damage to oral and dental tissues by inhibiting the expression of virulence traits

- Suppress the competitiveness of some of the putative pathogens by both restricting their growth and denying them the environment they need to flourish (for example, acidic conditions or presence of novel host proteins).

It has been shown that plaque biofilms need to be established for two days or longer before the $\mathrm{pH}$ gradients following sucrose metabolism fall below the critical $\mathrm{pH}$ for enamel demineralisation, ${ }^{25}$ that is, thinner biofilms are less damaging to the host than thicker and more mature biofilms. Thus, the action of oral care products can help to preserve an appropriate biofilm structure and promote the stability of the beneficial resident microflora (microbial homeostasis).

\section{CAN WE TRANSLATE THIS KNOWL- EDGE INTO CLINICAL PRACTICE?}

It has been argued in this article that the presence of biofilms in the mouth is both natural and is of benefit to the host. Therefore, there is a clear need to maintain these beneficial micro-organisms. Disease is due to imbalances in the proportions of this resident microflora driven by deleterious changes in local environmental conditions (the ecological plaque hypothesis). ${ }^{18,26}$ Briefly, poor oral hygiene can lead to an increase in plaque mass which, when coupled with a substantial change in environmental conditions in the mouth, can affect the competitiveness of plaque bacteria within the biofilm, leading to the enrichment of organisms most suited to the altered environment and result in a breakdown of microbial homeostasis
(Fig. 2). In caries, an increased frequency of sugar intake, or a reduction in saliva flow, results in plaque biofilms spending more time at low $\mathrm{pH}$. This selects for acid-producing and acid-tolerating species (most commonly mutans streptococci, but not exclusively so) at the expense of health-associated bacteria that prefer $\mathrm{pH}$ values around neutrality. Increases in the acidogenic populations lead to even more acid production and lower $\mathrm{pH}$ levels within the biofilm, which further disrupts microbial homeostasis and promotes demineralisation. ${ }^{18,26}$ In gingivitis, the inflammatory response to plaque accumulation results in an increased flow of GCF which, in addition to introducing components of the host defences, also delivers host molecules such as haemoglobin and transferrin that act as essential nutrients for many of the obligately anaerobic and proteolytic bacteria detected in higher proportions in periodontal disease. The metabolism of the subgingival microflora makes the site more anaerobic and the local $\mathrm{pH}$ increases due to proteolysis. These environmental changes drive the selection of the diverse microbial 
consortia that are detected at inflamed sites. ${ }^{18}$ A key principle of this hypothesis is that disease can be treated not only by (a) improving oral hygiene or (b) targeting the putative pathogens directly, but also by (c) interfering with the environmental pressures that select for the pathogenic microorganisms. ${ }^{18}$ Antimicrobial agents in oral care products can play an important role in all of these stages, ${ }^{27,28}$ for example, by killing some of the key bacteria and by reducing (at sub-lethal concentrations) the deleterious consequences to the host associated with acid production ${ }^{29-31}$ and proteolysis $^{29,32}$ that create the selection pressures for the overgrowth of putative pathogens in oral biofilms. ${ }^{23}$

The stratagem for using antimicrobial agents in oral care products, therefore, is quite distinct to that when prescribing antibiotics in clinical medicine. In cases of the latter, high doses of antibiotic (preferably with a bactericidal mode of action) are given for a fixed period with the intention of eliminating a recognised pathogen, often from a site that should be relatively sterile. In oral care, antimicrobial agents are delivered in over-the-counter products and used unsupervised, on a regular basis, at a site with a resident and beneficial microflora. Thus, it can be appreciated that agents that work subtly but effectively over long time periods to suppress or restrict the growth and metabolism of certain sections of the biofilm consortium may be ideal for the long-term control of oral biofilms. These oral care products help preserve the natural microbial composition and activity, as well as the important beneficial functions, of our resident oral microflora (Fig. 2), and in so doing, help reconcile the paradoxes described at the start of this article.

The author has received a fee from Johnson \& Johnson for writing this review article and in the past has acted as a consultant to, and received research grants from, several oral care companies.

1. Dewhirst FE, Chen T, Izard J et al. The human oral microbiome. J Bacterio/ 2010; 192: 5002-5017.

2. Wilson M. Microbial inhabitants of humans. Their ecology and role in health and disease. Cambridge: Cambridge University Press, 2005.

3. Jakubovics N S. Talk of the town: interspecies communication in oral biofilms. Mol Oral Microbiol 2010; 25: 4-14.

4. Marsh P D. Dental plaque: biological significance of a biofilm and community life-style. J Clin Periodontol 2005; 32: 7-15.

5. Wilks M. Bacteria and early human development Early Hum Dev 2007; 83: 165-170.

6. Marsh P D. Role of the oral microflora in health. Microb Ecol Health D 2000; 12: 130-137.

7. Srinivasan N. Telling apart friend from foe: discriminating between commensals and pathogens at mucosal sites. Innate Immun 2010; 16: 391-404.

8. Cosseau C, Devine D A, Dullaghan E et al. The commensal Streptococcus salivarius K12 downregulates the innate immune responses of human epithelial cells and promotes host-microbe homeostasis. Infect Immun 2008; 76: 4,163-4,175.

9. Milward M R, Chapple I L, Wright H J, Millard J L, Matthews J B, Cooper P R. Differential activation of NF-kappaB and gene expression in oral epithelial cells by periodontal pathogens. Clin Exp Immunol 2007; 148: 307-324.

10. Govoni M, Jansson E A, Weitzberg E, Lundberg J 0 The increase in plasma nitrite after a dietary nitrate load is markedly attenuated by an antibacterial mouthwash. Nitric Oxide 2008; 19: 333-337.

11. Petersson J, Carlström M, Schreiber 0 et al. Gastroprotective and blood pressure lowering effects of dietary nitrate are abolished by an antiseptic mouthwash. Free Radic Biol Med 2009; 46: 1068-1075

12. Dougall H T, Smith L, Duncan C, Benjamin N. The effect of amoxycillin on salivary nitrite concentrations: an important mechanism of adverse reactions? Br J Clin Pharmacol 1995; 39: 460-462.

13. Herrera $D$, Alonso $B$, León $R$, Roldán $S$, Sanz $M$. Antimicrobial therapy in periodontitis: the use of systemic antimicrobials against the subgingival biofilm. J Clin Periodonto/ 2008; 35: 45-66.

14. Wade W G. Has the use of molecular methods for the characterization of the human oral microbiome changed our understanding of the role of bacteria in the pathogenesis of periodontal disease? J Clin
Periodonto/ 2011; 38: 7-16.

15. Aas J A, Paster B J, Stokes L N, Olsen I, Dewhirst $F$ E. Defining the normal bacterial flora of the oral cavity. J Clin Microbiol 2005; 43: 5721-5732.

16. Marsh P D, Martin M V. Oral Microbiology. 5th ed. Edinburgh: Churchill Livingstone Elsevier, 2009.

17. Marsh P D. Host defenses and microbial homeostasis: role of microbial interactions. J Dent Res 1989; 68: 1567-1575.

18. Marsh P D. Are dental diseases examples of ecological catastrophes? Microbiology 2003; 149: 279-294.

19. Scully C, Greenman J. Halitosis (breath odour) Periodontol 2000 2008; 48: 66-75.

20. Hughes FJ, McNab R. Oral malodour - a review. Arch Oral Biol 2008; 53 (Suppl 1): S1-7.

21. Scannapieco FA, Dasanayake A P, Chhun N. Does periodontal therapy reduce the risk for systemic diseases? Dent Clin North Am 2010; 54: 163-181.

22. Paju S, Scannapieco F A. Oral biofilms, periodontitis, and pulmonary infections. Oral Dis 2007; 13: 508-512.

23. Marsh P D. Controlling the oral biofilm with antimicrobials. J Dent 2010; 38 (Suppl 1): S11-15.

24. Bradshaw D J, Marsh P D, Watson G K, Cummins D. The effects of triclosan and zinc citrate, alone and in combination, on a community of oral bacteria grown in vitro. J Dent Res 1993; 72: 25-30.

25. Imfeld T N, Lutz F. Intraplaque acid formation assessed in vivo in children and young adults. Pediatr Dent 1980; 2: 87-93.

26. Takahashi N, Nyvad B. Caries ecology revisited: microbial dynamics and the caries process. Caries Res 2008; 42: 409-418.

27. Cummins D. Mechanisms of action of clinically proven antiplaque agents. In Embery G, Rolla G (eds). Clinical and biological aspects of dentifrices. pp 205-228. Oxford: Oxford University Press, 1992.

28. Scheie A A, Petersen F C. Antimicrobials in caries control. In Fejerskov 0, Kidd E A M (eds). Dental caries: the disease and its clinical management. 2nd ed. pp 265-277. Oxford: Blackwell Munksgaard, 2008.

29. Rogers A H, Zilm PS, Gully N J, Pfennig A L Chlorhexidine affects arginine metabolism as well as glycolysis in a strain of Streptococcus sanguis. Oral Microbiol Immunol 1987: 2: 172-182.

30. Wikén Albertsson $K$, Persson A, Lingström P, van Dijken J W. Effects of mouthrinses containing essential oils and alcohol-free chlorhexidine on human plaque acidogenicity. Clin Oral Investig 2010; 14: 107-112.

31. Zhang J Z, Harper D S, Vogel G L, Schumacher G. Effect of an essential oil mouthrinse, with and without fluoride, on plaque metabolic acid production and $\mathrm{pH}$ after a sucrose challenge. Caries Res 2004; 38: 537-541.

32. Cummins D. Zinc citrate/Triclosan: a new anti-plaque system for the control of plaque and the prevention of gingivitis: short-term clinical and mode of action studies. J Clin Periodontol 1991; 18: 455-461. 